\title{
Dynamic Analysis of Mindlin Plates Resting on a Viscoelastic Foundation Subjected to Moving Loads During Abrupt Braking using Moving Element Method
}

\author{
Cao Tan Ngoc Than ${ }^{1}$, Luong Van Hai ${ }^{1, *}$, Nguyen Xuan Vu ${ }^{1}$, Tran Minh Thi ${ }^{1}$ \\ ${ }^{I}$ Department of Civil Engineering, Ho Chi Minh University of Technology, Vietnam National University Ho Chi Minh \\ City (VNU-HCM), Vietnam \\ *Corresponding author: lvhai@hcmut.edu.vn
}

\begin{abstract}
The paper proposes a new computational approach using the moving element method (MEM) for simulating the dynamic responses of Mindlin plate resting on a viscoelastic foundation subjected to moving loads during abrupt braking. In this approach, the governing equations as well as the plate element mass, damping and stiffness matrices are formulated in a convected coordinate in which the origin is attached to the applied point of the moving load. Thus, the proposed method simply treats the moving loads as 'stationary' at the nodes of the plate to avoid updating the locations of moving loads due to the change of the contact points on the plate. The interaction between the moving load and the plate during abrupt braking is accounted for through the vertical force and tangential wheel-pavement friction force. The effects of wheel sliding, load deceleration magnitude, friction coefficient, and plate thickness on the dynamic responses of plate are investigated.
\end{abstract}

Keywords—Abrupt braking; Dynamic responses; Mindlin plates; Moving element method (MEM); Wheel sliding.

\section{INTRODUCTION}

$\mathrm{T}_{\mathrm{h}}$ dynamic responses of plates resting on a viscoelastic foundation subjected to moving loads have attracted much research attention in the last two decades. The results of these studies can be employed in many branches of modern transportation engineering such as the design of track/road beds, highway and runway pavements. Analytical methods were used in earlier research works to study the static and dynamic responses of plates. A few of these works related to the dynamic analysis of plates resting on a viscoelastic foundation subjected to moving loads are those works by Gbadeyan and Oni [1], Kim and Reosset [2], Huang and Thambiratnam [3],[4], Sun[5],[6].

The Finite Element Method (FEM) is a wellestablished numerical method widely used to solve many complicated problems, including problems involving moving loads. Wu et al. [7] used the FEM to analyze the responses of flat plate subjected to various moving loads. The effects of eccentricity, acceleration and initial velocity of moving loads and the effects of span length on the dynamic responses of plates were examined in this study. Zaman et al. [8] investigated the dynamic responses of a thick plate on viscoelastic foundation subjected to moving loads by taking into account the transverse shear deformation as well as the bending of the slab. Phung-Van et al. [9] developed the CS-DSG3 for analyzing dynamic responses of Mindlin and composite plates on the viscoelastic foundation subjected to moving loads. Li et al. [10] studied the dynamic responses of a rectangular plate on a viscoelastic foundation subjected to moving loads with varying velocity. Three types of moving loads, including acceleration, deceleration and uniform speed were discussed.

In studies using the FEM, a global coordinate system fixed in space is normally defined to form the structural matrices. The complication arises as the load moves from one finite element into another element, and hence the load position has to be updated at every time step of the solution procedure. In addition, in the case of infinitely long plates subjected to moving loads such as the pavement of highway or runway, some additional boundary conditions have to be introduced artificially to truncate the infinitely long plate at the edges of the plate. In those cases, the moving loads will soon approach closely to the artificial boundary end on the 'downstream' side and may even go beyond the downstream end. This difficulty can be overcome by employing a large enough domain size but the computational cost will increase significantly.

To overcome such the complication encountered in the FEM, Krenk et al. [11] proposed the use of FEM in convected coordinates to obtain the response of an elastic half-space subjected to a moving load. This approach will help overcome the problem related to the moving loads travelling over a finite domain. Andersen et al. [12] gave a formulation in convected coordinates for the problem of a beam on a Kelvin foundation subjected to a harmonic moving load. Koh et al. [13] adopted the idea of convected coordinates for solving train-track problems, and named the numerical algorithm as Moving Element Method (MEM). The method was subsequently applied to the analysis of in-plane dynamic responses of annular disk (Koh et al. [14]) and moving load on continuum (Koh et al. [15]). Xu et al. [16] extended the one-dimensional MEM proposed by Koh et al. [13] to two dimensional problems in which the vehicle moves on an infinite Kirchhoff plate supported by Kelvin 
foundation. Recently, Ang and Dai [17] employed the MEM computational to investigate the dynamic response of high-speed rail system for the situation where there is an abrupt change of foundation stiffness. The influence of different factors, including the degree of change of foundation stiffness, traveling velocity of the train and the severity of track irregularity, on the response of the train as well as the track is examined and discussed. Ang et al. [18] applied the MEM to investigate the "jumping wheel" phenomenon in high-speed train motion at constant velocity over a transition region where there is a sudden change of the foundation stiffness. Tran et al. [19] analyzed the dynamic response of high-speed rail (HSR) traveling at non-uniform speed using the MEM. In this study, both linear and nonlinear wheel-rail contact models were examined. Dai and Ang [20] presented an analytical solution to the steady-state response of a curved beam resting on a viscously damped foundation and subjected to a single or sequence of moving loads by using MEM. On the same thread, Tran et al. [21], [22], [23][24] employed the MEM to investigate the dynamic response of high-speed rail experiencing heavy braking and abrupt braking. The effects of the wheel sliding, initial train deceleration, initial train speed and the severity of the railhead roughness on the dynamic response of the HSR were investigated.

In the aforementioned studies, the MEM has been successfully applied to the analyses of various moving load problems including the train-track dynamics. However, such suprior approach has rarely been applied to the analysis of plates under moving loads. To the author's knowledge, no studies have investigated the dynamic responses of plate subjected to moving loads during abrupt braking.

Hence in order to fill in the abovementioned research gap, the present paper proposes a computational approach using the MEM for analyzing the dynamic responses of Mindlin plate resting on a viscoelastic foundation subjected to moving loads during abrupt braking. In this approach, the plate is truncated and discretized into "moving-elements", which are not physical elements fixed to the plate but are conceptual elements that "flow" with the moving load along the plate. The formulations of plate element mass, damping and stiffness matrices, which are written in a relative coordinate attached to the moving load, are derived. The interaction between the moving load and the plate during abrupt braking is accounted for through the vertical force and tangential wheel-pavement friction forces. To verify responses of plates resting on a viscoelastic foundation subjected to moving loads are examined. The results obtained in this study are compared with other published results in the literature. Next, the effects of wheel sliding, load deceleration magnitude, friction coefficient, and plate thickness on the dynamic responses of plate are investigated.

\section{FORMULATION AND METODOLOGY}

\section{A. Weakform for the Mindlin plate resisting on viscoelastic foundation}

Let us consider an isotropic Mindlin plate with length $L$, width $B$, thickness $h$ resting on a viscoelastic foundation under bending deformation as shown in Figure 1. The viscoelastic foundation has foundation stiffness $k_{f}$ and foundation damping coefficient $c_{f}$. The middle (neutral) surface of the plate is chosen as the reference plane that occupies a domain $\Omega \subset R^{2}$. Let $w$ be the vertical deflection and $\boldsymbol{\beta}=\left[\begin{array}{ll}\beta_{x} & \beta_{y}\end{array}\right]^{T}$ be the vector of rotations, in which $\beta_{x}, \beta_{y}$ are the rotations of the middle plane around $y$-axis and $x$-axis, respectively, with the positive directions defined in Figure 1.

The unknown vector of three independent field variables at any point in the middle plane of the plate can be written as

$$
\mathbf{u}=\left[\begin{array}{lll}
w & \beta_{x} & \beta_{y}
\end{array}\right]^{T}
$$

The curvature of the deflected plate $\boldsymbol{\kappa}$ and the shear strains $\boldsymbol{\gamma}$ are defined, respectively, as

$$
\begin{gathered}
\mathbf{\kappa}=\mathbf{L}_{d} \boldsymbol{\beta} \\
\boldsymbol{\gamma}=\nabla w+\boldsymbol{\beta}
\end{gathered}
$$

where $\nabla=\left[\begin{array}{ll}\partial / \partial x & \partial / \partial y\end{array}\right]^{T}$, and $\mathbf{L}_{d}$ is a differential operator matrix defined by

$$
\mathbf{L}_{d}=\left[\begin{array}{cc}
\partial / \partial x & 0 \\
0 & \partial / \partial y \\
\partial / \partial y & \partial / \partial x
\end{array}\right]
$$

The Galerkin weak form for the dynamic equilibrium equation of the Mindlin plates resting on a viscoelastic foundation can be written as

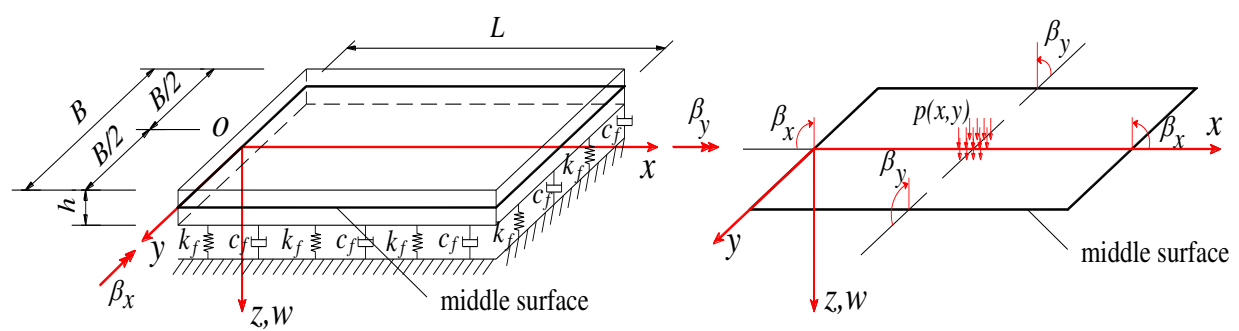

Figure 1. Model of a Mindlin plate resting on a viscoelastic foundation and positive directions of vertical displacement $w$ and two rotations $\beta_{x}, \beta_{y}$.

the accuracy of the proposed method, the dynamic 
produced between the $i$ th wheel of the vehicle and the pavement may be written as

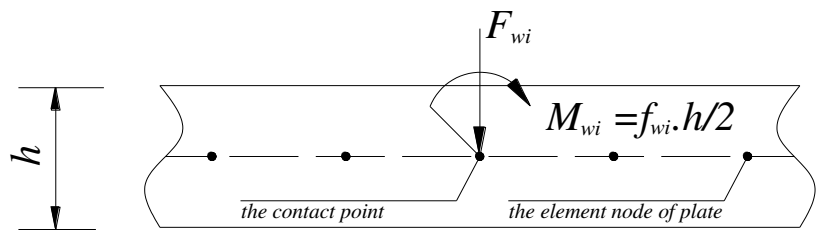

Figure 2. The model of the friction force acting on the pavement.

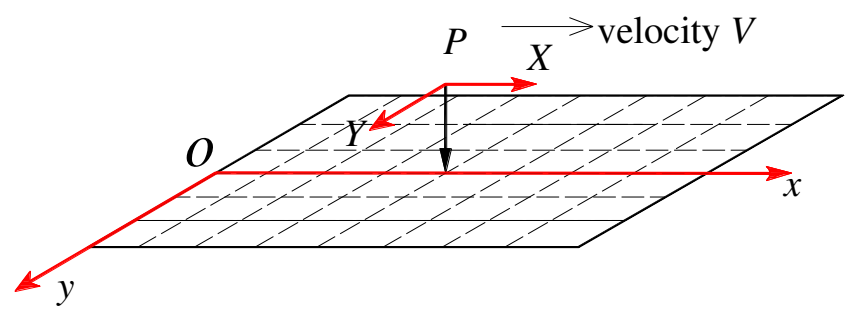

Figure 3. Discretization of a plate into $N_{e}$ moving elements.

$$
\begin{aligned}
& \int_{\Omega} \delta \kappa^{T} \mathbf{D}_{b} \kappa \mathrm{d} \Omega+\int_{\Omega} \delta \gamma^{T} \mathbf{D}_{s} \gamma \mathrm{d} \Omega+\int_{\Omega} \delta \mathbf{u}^{T} \mathbf{m u ̈} \mathrm{d} \Omega+ \\
& \int_{\Omega} \delta w^{T} k_{f} w \mathrm{~d} \Omega+\int_{\Omega} \delta w^{T} c_{f} \dot{w} \mathrm{~d} \Omega=\int_{\Omega} \delta \mathbf{u}^{T} \mathbf{b} \mathrm{d} \Omega
\end{aligned}
$$

where $\mathbf{b}=\left[\begin{array}{lll}p(x, y) & 0 & 0\end{array}\right]^{T}$ is the vector of the distributed load $p(x, y)$ applied on the plate; $\mathbf{m}$ is the mass matrix containing the mass density of the material $\rho$ and plate thickness $h$

$$
\mathbf{m}=\rho \operatorname{diag}\left[h, \frac{h^{3}}{12}, \frac{h^{3}}{12}\right]
$$

and $\mathbf{D}_{b}, \mathbf{D}_{s}$ are the material matrices related to the flexural and shear rigidity, respectively, and are given by in which $E$ is the Young's modulus; $v$ is poison's ratio; $k=5 / 6$ is shear correction factor.

$$
\mathbf{D}_{b}=\frac{E h^{3}}{\left(1-v^{2}\right)}\left[\begin{array}{ccc}
1 & v & 0 \\
v & 1 & 0 \\
0 & 0 & \frac{1-v}{2}
\end{array}\right] ; \mathbf{D}_{s}=\frac{E k h}{2(1+v)}\left[\begin{array}{ll}
1 & 0 \\
0 & 1
\end{array}\right]
$$

in which $E$ is the Young's modulus; $v$ is poison's ratio; $k=5 / 6$ is shear correction factor.

\section{B. The friction force acting on the pavement}

The vehicle is assumed to be traveling at an initial constant velocity and the vehicle brakes are then suddenly applied at a certain instant of time. Depending on the coefficient of static friction between the wheels and the pavement, sliding of wheels may subsequently occur. The friction forces produced between the wheels and the pavement depend on whether sliding of wheels occurs. Sliding occurs when and vice versa, where $\mathrm{H}$ denotes the total horizontal inertial force, $\mathrm{N}$ the total vertical contact force and the coefficient of static friction between wheel and pavement. The friction force

$$
\begin{aligned}
& f_{i}= \begin{cases}\frac{H}{N} F_{w i} & \text { for } H \leq \mu_{s} N \\
\mu_{k} F_{w i} & \text { for } H>\mu_{s} N\end{cases} \\
& H=m_{v e} a_{d e} \\
& N=\sum_{i=1}^{n} F_{w i}
\end{aligned}
$$

in which $m_{v e}$ is the weight of the vehicle; $a_{d e}$ is the vehicle deceleration; $F_{w i}$ is the vertical contact force at the $i$ th wheel; $\mu_{k}$ is the coefficient of kinetic friction between the wheel and the pavement.

In case of a concentrated load $P$ moving on the plate, the vertical contact force $F_{w i}$ at the $i$ th wheel and the total vertical contact force $N$ is assumed as

$$
N=F_{w i}=P
$$

Substituting Eq. (11)into Eq. (8), the magnitude of the vehicle deceleration which causes the sliding of wheels is given as

$$
a_{d e} \geq \mu_{s} g
$$

Figure 2 shows the model of the friction force acting on the pavement. In this study, the effect of the axial force on the dynamic response of the plate is neglected. The friction force acting on the pavement is modeled as a concentrated moment $M_{\mathrm{wi}}$ at the contact point and defined as

$$
M_{\mathrm{w} i}=f_{\mathrm{w} i} \frac{h}{2}
$$

In view of moving load problem, the plate is subjected to a concentrated load $P$ moving along the midline in $x$-direction and experienced to abrupt braking. The load vector $\mathbf{b}$ in Eq. (5) is rewritten as $\mathbf{b}=\left[\begin{array}{lll}P \delta(x-s) \delta(y-0) & f_{w i} \frac{h}{2} \delta(x-s) \delta(y-0) & 0\end{array}\right]^{T}$ 
where $S$ is the distance traveled by the load at any instant $t ; \delta$ denotes the Dirac-delta function that equals

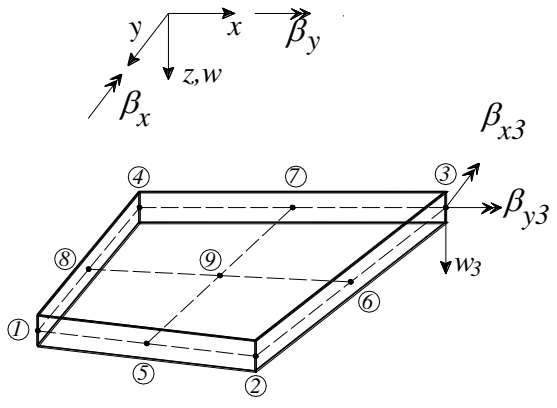

(a)
By means of the FEM, the quadrilateral nine-node element, $Q 9$, of the serendipity family is employed in this numerical model to ensure both the deflection and

Figure 4. The quadrilateral nine-node elements, Q9, of the serendipity family: (a) Q9 element in global coordinates $(x, y)$; (b) Q9 element in natural coordinates $(\xi, \eta)$.

unity at $x=s$ and $y=0$, and zero elsewhere; $t$ is the travel time of the moving load.

C. Moving element method for the Mindlin plate resting on a viscoelastic foundation subjected to moving load

In this paper, the one-dimensional MEM proposed by Koh et al. [13] is extended to solve the two dimensional problems of the Mindlin plate resting on the viscoelastic foundation subjected to moving load. The bounded domain $\Omega$ of the plate is discretized into $N_{e}$ moving elements such that $\Omega=\bigcup_{e=1}^{N_{e}} \Omega_{e}$ and $\Omega_{i} \cap \Omega_{j} \neq \varnothing$, $i \neq j$ as shown in Figure 3 .

In view of the moving element method (MEM), a moving coordinates system $(X, Y)$ is attached to the applied point of the moving load $P$ and moves at the same speed as the moving load. The relationship between the moving coordinate $(X, Y)$ and the fixed coordinate $(x, y)$ is given by

$$
\begin{aligned}
& X=x-s \\
& Y=y \\
& s=V_{o} t+\frac{1}{2} a t^{2} \\
& V=V_{o}+a t
\end{aligned}
$$

where $V_{o}, V, a$ are the initial velocity, the velocity at any instant $t$, the acceleration/deceleration of the moving load, respectively.

In view of Eq. (15), Eq. (5) is rewritten as

$$
\begin{aligned}
& \int_{\Omega} \delta \boldsymbol{\kappa}^{T} \mathbf{D}_{b} \mathbf{\kappa} \mathrm{d} \Omega+\int_{\Omega} \delta \gamma^{T} \mathbf{D}_{s} \gamma \mathrm{d} \Omega+\int_{\Omega} \delta \mathbf{u}^{T} \mathbf{m}\left(V^{2} \frac{\partial^{2} \mathbf{u}(r, t)}{\partial r^{2}}\right. \\
& \left.-2 V \frac{\partial^{2} \mathbf{u}(r, t)}{\partial r \partial t}-a \frac{\partial \mathbf{u}(r, t)}{\partial r}+\frac{\partial^{2} \mathbf{u}(r, t)}{\partial t^{2}}\right) \mathrm{d} \Omega \\
& +\int_{\Omega} \delta w^{T} k_{f} w \mathrm{~d} \Omega+\int_{\Omega} \delta w^{T} c_{f}\left(-V \frac{\partial w(r, t)}{\partial r}+\frac{\partial w(r, t)}{\partial t}\right) \mathrm{d} \Omega=\int_{\Omega} \delta \mathbf{u}^{T} \mathbf{b} \mathrm{d} \Omega
\end{aligned}
$$

slope compatibility on the adjacent elements. Figure 4(a) and Figure 4(b) show the $Q 9$ elements in the global coordinates $(x, y)$ system and in the natural coordinates $(\xi, \eta)$ system.

All $Q 9$ elements are transformed from the global coordinates $(x, y)$ into the natural coordinates $(\xi, \eta)$. The shape functions for the $Q 9$ element in the natural coordinates $(\xi, \eta)$ can be derived as

$N_{1}=\frac{1}{4}(\xi-1)(\eta-1) \xi \eta, \quad N_{2}=\frac{1}{4}(\xi+1)(\eta-1) \xi \eta, \quad N_{3}=\frac{1}{4}(\xi+1)(\eta+1) \xi \eta$,

$N_{4}=\frac{1}{4}(\xi-1)(\eta+1) \xi \eta, \quad N_{5}=\frac{1}{2}\left(1-\xi^{2}\right)(\eta-1) \eta, \quad N_{6}=\frac{1}{2}\left(1-\eta^{2}\right)(\xi+1) \xi$

$N_{7}=\frac{1}{2}\left(1-\xi^{2}\right)(\eta+1) \eta, \quad N_{8}=\frac{1}{2}\left(1-\eta^{2}\right)(\xi-1) \xi, \quad N_{9}=\left(1-\xi^{2}\right)\left(1-\eta^{2}\right)$

Using the shape functions, the displacement vector $\mathbf{u}=\left[\begin{array}{lll}w & \beta_{x} & \beta_{y}\end{array}\right]^{T}$ and the vertical displacement $w$ within each element can be interpolated from the nodal displacements of the element, respectively, as

$$
\begin{gathered}
\mathbf{u}=\mathbf{N} \mathbf{d}_{e} \\
w=\mathbf{N}_{w} \mathbf{d}_{e}
\end{gathered}
$$

where $\mathbf{N}, \mathbf{N}_{w}$ are the matrices containing the shape functions as

$$
\begin{aligned}
& \mathbf{N}=\left[\begin{array}{cccccccccc}
N_{1} & 0 & 0 & N_{2} & 0 & 0 & \ldots & N_{9} & 0 & 0 \\
0 & N_{1} & 0 & 0 & N_{2} & 0 & \ldots & 0 & N_{9} & 0 \\
0 & 0 & N_{1} & 0 & 0 & N_{2} & \ldots & 0 & 0 & N_{9}
\end{array}\right] \\
& \mathbf{N}_{w}=\left[\begin{array}{llllllllll}
N_{1} & 0 & 0 & N_{2} & 0 & 0 & \ldots & N_{9} & 0 & 0
\end{array}\right]
\end{aligned}
$$

and $\mathbf{d}_{e}$ is the node displacement vector of the element as

$$
\mathbf{d}_{e}=\left[\begin{array}{llllllllll}
w_{1} & \beta_{x 1} & \beta_{y 1} & w_{2} & \beta_{x 2} & \beta_{y 2} & \ldots & w_{9} & \beta_{x 9} & \beta_{y 9}
\end{array}\right]^{T}
$$

The bending and shear strains can be expressed in the matrix forms as

$$
\mathbf{\kappa}=\mathbf{B}_{b} \mathbf{d}_{e}, \boldsymbol{\gamma}=\mathbf{B}_{s} \mathbf{d}_{e}
$$


where $\ddot{\mathbf{d}}, \dot{\mathbf{d}}$ and $\mathbf{d}$ denote the global acceleration, global velocity and global displacement vectors of the nodal, respectively; $\mathbf{M}, \mathbf{C}$ and $\mathbf{K}$ the global mass, global damping and global stiffness matrices, respectively; and

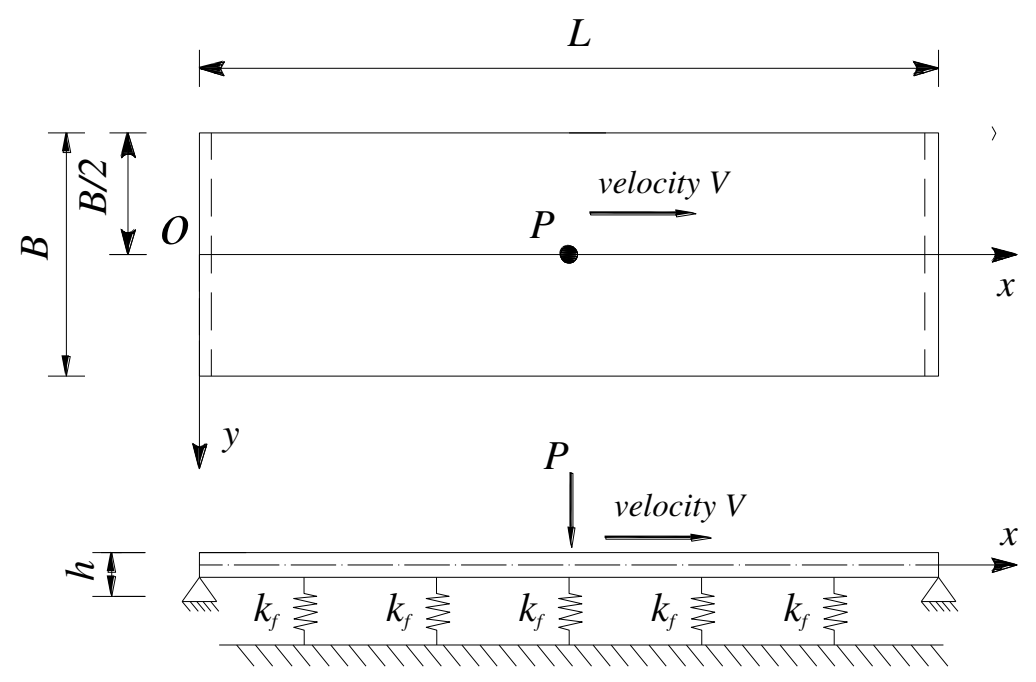

Figure 5. Model of a plate resting on an elastic foundation subjected to a moving load.

$$
\begin{gathered}
\mathbf{B}_{b}=\left[\begin{array}{cccccccccc}
0 & \frac{\partial N_{1}}{\partial x} & 0 & 0 & \frac{\partial N_{2}}{\partial x} & 0 & \ldots & 0 & \frac{\partial N_{9}}{\partial x} & 0 \\
0 & 0 & \frac{\partial N_{1}}{\partial y} & 0 & 0 & \frac{\partial N_{2}}{\partial y} & \ldots & 0 & 0 & \frac{\partial N_{9}}{\partial y} \\
0 & \frac{\partial N_{1}}{\partial y} & \frac{\partial N_{1}}{\partial x} & 0 & \frac{\partial N_{2}}{\partial y} & \frac{\partial N_{2}}{\partial x} & \ldots & 0 & \frac{\partial N_{9}}{\partial y} & \frac{\partial N_{9}}{\partial x}
\end{array}\right] \\
\mathbf{B}_{s}=\left[\begin{array}{cccccccccc}
\frac{\partial N_{1}}{\partial x} & N_{1} & 0 & \frac{\partial N_{2}}{\partial x} & N_{2} & 0 & \ldots & \frac{\partial N_{9}}{\partial x} & N_{9} & 0 \\
\frac{\partial N_{1}}{\partial y} & 0 & N_{1} & \frac{\partial N_{2}}{\partial y} & 0 & N_{2} & \ldots & \frac{\partial N_{9}}{\partial y} & 0 & N_{9}
\end{array}\right]
\end{gathered}
$$

Substituting Eq. (18), Eq. (19)and Eq. (23) into Eq. (16) and using simple rearrangement, the Galerkin weakform for the Mindlin plate element can be expressed as

$$
\begin{aligned}
& \left(\begin{array}{l}
\int_{\Omega_{e}} \mathbf{B}_{b}^{T} \mathbf{D}_{b} \mathbf{B}_{b} \mathrm{~d} \Omega_{e}+\int_{\Omega_{e}} \mathbf{B}_{s}^{T} \mathbf{D}_{s} \mathbf{B}_{s} \mathrm{~d} \Omega_{e}+\mathbf{m} V^{2} \int_{\Omega_{e}} \mathbf{N}^{T} \mathbf{N}_{, r r} \mathrm{~d} \Omega_{e}+k_{f} \int \mathbf{N}_{\Omega_{e}}^{T} \mathbf{N}_{w} \mathrm{~d} \Omega_{e} \\
-c_{f} V \int_{\Omega_{e}} \mathbf{N}_{w}^{T} \mathbf{N}_{w, r} \mathrm{~d} \Omega_{e}-\mathbf{m} a \int_{\Omega_{e}} \mathbf{N}^{T} \mathbf{N}_{, r} \mathrm{~d} \Omega_{e}
\end{array}\right) \mathbf{d}_{e} \\
& +\left(-2 \mathbf{m} V \int_{\Omega_{e}} \mathbf{N}^{T} \mathbf{N}_{, r} \mathrm{~d} \Omega_{e}+c_{f} \int \mathbf{N}_{\Omega_{e}}^{T} \mathbf{N}_{w} \mathrm{~d} \Omega_{e}\right) \dot{\mathbf{d}}_{e}+\left(\mathbf{m} \int_{\Omega_{e}} \mathbf{N}^{T} \mathbf{N} \mathbf{d} \Omega_{e}\right) \ddot{\mathbf{d}}_{e}=\int_{\Omega_{e}} \mathbf{N}^{T} \mathbf{b} \mathrm{d} \Omega_{e}
\end{aligned}
$$

The element mass, damping and stiffness matrices in the moving coordinate system can be expressed as

$$
\begin{gathered}
\mathbf{M}_{e}=\mathbf{m} \int \mathbf{N}^{T} \mathbf{N} \mathrm{d} e \\
\mathbf{C}_{e}=2 \mathbf{m} V \int \mathbf{N}^{T} \mathbf{N}_{, r} \mathrm{~d} e_{e}+C_{f} \int \mathbf{N}_{w}^{T} \mathbf{N}_{w} \mathrm{~d} e \\
\mathbf{K}_{e}=\int_{\Omega_{e}} \mathbf{B}_{b}^{T} \mathbf{D}_{b} \mathbf{B}_{b} \mathrm{~d} \Omega_{e}+\int_{\Omega_{e}} \mathbf{B}_{s}^{T} \mathbf{D}_{s} \mathbf{B}_{s} \mathrm{~d} \Omega_{e}+\mathbf{m} V^{2} \int_{\Omega_{e}} \mathbf{N}^{T} \mathbf{N}_{, r} \mathrm{~d} \Omega_{e} \\
+k_{f} \int_{\Omega_{e}} \mathbf{N}_{w}^{T} \mathbf{N}_{w} \mathrm{~d} \Omega_{e}-c_{f} V \int_{\Omega_{e}} \mathbf{N}_{w}^{T} \mathbf{N}_{w, r} \mathrm{~d} \Omega_{e}-\mathbf{m} a \int_{\Omega_{e}} \mathbf{N}^{T} \mathbf{N}_{, r} \mathrm{~d} \Omega_{e}
\end{gathered}
$$

where ()$_{, r},()_{, r r}$ denote partial derivative and second partial derivative with respect to $r$, respectively.

Assembling all element matrices gives the equation of the motion of the plate as

$$
\mathbf{M} \ddot{d}+\mathbf{C} \dot{d}+\mathbf{K d}=\mathbf{P}
$$

$\mathbf{P}$ the global load vector. The above dynamic equation can be solved by any direct integration methods such as Newmark- $\beta$ method.

\section{NUMERICAL RESULTS}

In this section, several numerical examples are carried out in order to illustrate the performance of the MEM for the dynamic analysis of Mindlin plates resting on a viscoelastic foundation subjected to moving load during abrupt braking. The first example considers the dynamic responses of a Mindlin plate resting on an elastic foundation subjected to a moving load to verify the accuracy of the proposed method. The present results are compared with other published results in the literature. Next, the dynamic responses of plates resting on a viscoelastic foundation subjected to a moving load during abrupt braking are examined. The effects of wheel sliding, load deceleration magnitude, friction coefficient, and plate thickness on the dynamic responses of plate are investigated. In all numerical examples, the material properties of the plate are Young's modulus $E=3.1 \times 10^{10} \mathrm{~N} / \mathrm{m}^{2}$, Poisson's ratio $v=0.25$ and mass density $\rho=2440 \mathrm{~kg} / \mathrm{m}^{3}$ (Huang and Thambiratnam [4]).

\section{A. Verification}

For the purpose of comparison, we now consider a rectangular plate resting on an elastic foundation as shown in Figure 5. The plate has the dimensions of length $L=100 \mathrm{~m}$, width $B=10 \mathrm{~m}$, thickness $h=0.3$ $\mathrm{m}$, and rests on an elastic foundation with the stiffness $k_{f}=1 \times 10^{7} \mathrm{~N} / \mathrm{m}^{3}$. A load $P=1000 \mathrm{~N}$ moves along the longitudinal midline of the plate with constant velocity $V=20 \mathrm{~m} / \mathrm{s}$. The shorter edges of the plate $(x=0$ and $x=$ $100 \mathrm{~m})$ are simply supported while the remaining edges are free. In this analysis, the plate is discretized into 
$100 \times 10$ moving elements of size $1 \mathrm{mx} 1 \mathrm{~m}$. The equations of motion are solved using Newmark's constant acceleration method employing a time-step of

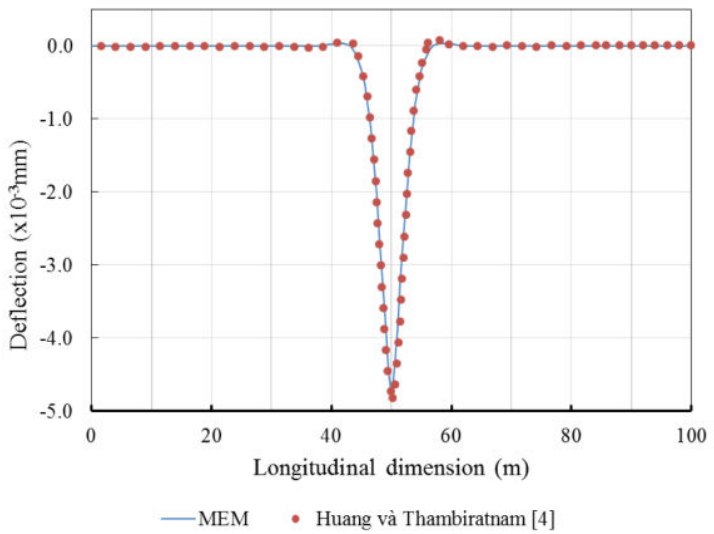

Figure 6. Deflected shapes along longitudinal midline of the plate when the moving load is applied at $x=50 \mathrm{~m}$.

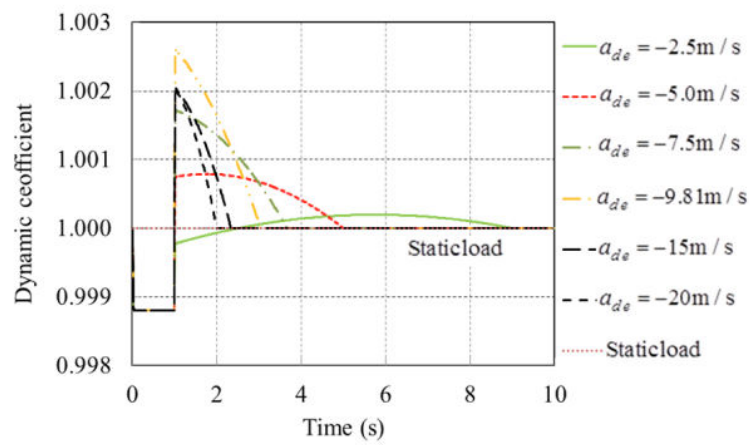

Figure 8. Effects of the load deceleration magnitude on the dynamic coefficient
Firstly, the effects of load deceleration on the dynamic responses of plates resting on a viscoelastic foundation are examined. The plate has the dimensions given by

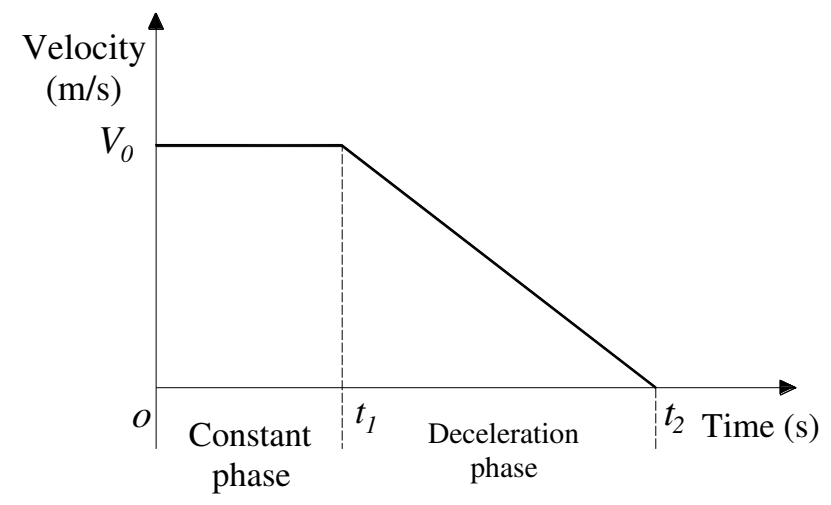

Figure 7. Load speed profile

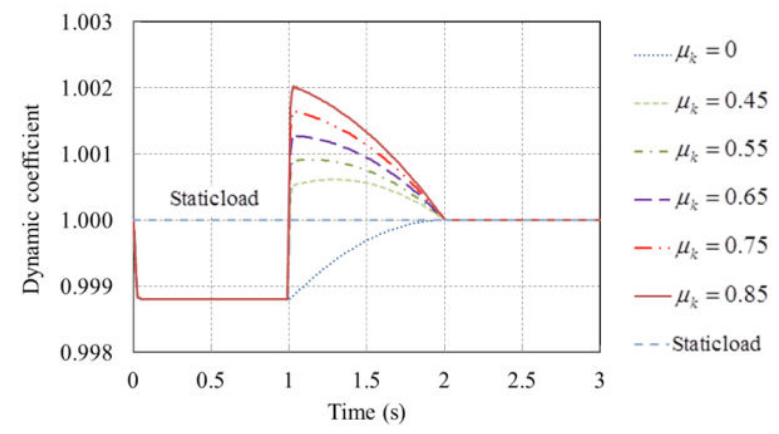

Figure 9. Effects of the kinetic friction coefficient on the dynamic coefficient

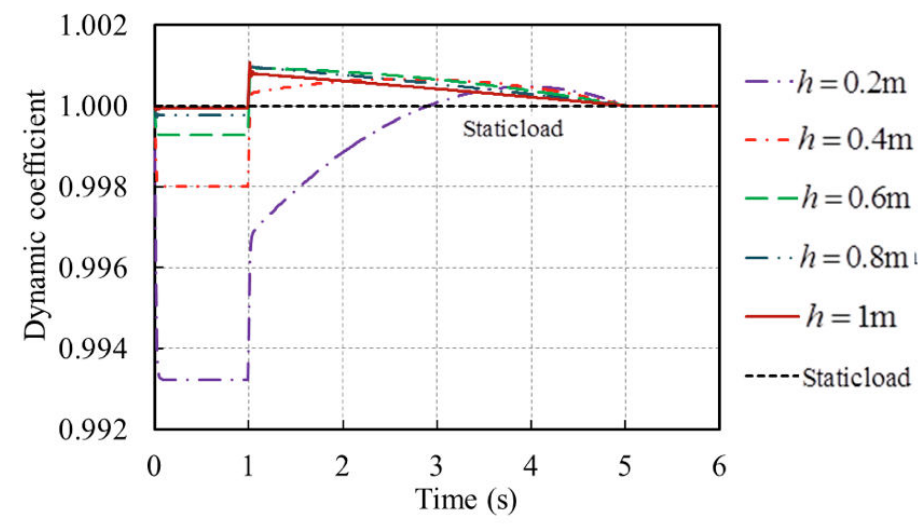

Figure 10. Effect of the plate thickness on the dynamic coefficient

0.0025s. Note that the MEM mesh and the time-step size are chosen based on the outcome of a convergence study.

Figure 6 shows the comparison of the deflected shape along longitudinal midline of the plate obtained in this study with that obtained by Huang and Thambiratnam [4]. The comparison shows that these results agree well with each other and the accuracy of the proposed MEM approach has been carried out.

B. Dynamic responses of plates subjected to moving load during abrupt braking length $L=30 \mathrm{~m}$, width $B=10 \mathrm{~m}$, thickness $h=0.5 \mathrm{~m}$, and rests on a viscoelastic foundation having stiffness $k_{f}=9.5 \times 10^{7} \mathrm{~N} / \mathrm{m}^{3}$ and damping coefficient $c_{f}=1 \times 10^{6}$ Ns $/ \mathrm{m}^{3}$. A concentrated load $P=83333 \mathrm{~N}$ (approximately the rear wheel load of the Dong Feng EQ1168G1 truck Zhao et al. [25]) moves along the longitudinal midline of the plate. The load is assumed to be traveling initially at a constant speed $V_{0}=20 \mathrm{~m} / \mathrm{s}$.

After time $t_{1}$, which is taken to be long enough for the vibration of the plate to attain steady-state, the load is 
then assumed to decelerate uniformly at $a_{d e}$ and finally comes to a halt at time $t_{2}$ as shown in Figure 7 . The coefficient of friction between the wheel and pavement depends on the pair of surfaces in contact and needs to be measured experimentally. For concrete and rubber, the coefficient of static friction, $\mu_{s}$, ranges typically from 0.3 (wet) to 1 (dry). The coefficient of kinetic friction, $\mu_{k}$, ranges from 0.45 (wet) to 0.85 (dry) [26].

It is of great interest to investigate the maximum central displacement of the plate subject to a moving load during abrupt braking. Let $w_{\text {centralstatic }}$ be the maximum central displacement of the plate when the moving load is a static load at the center of the plate and $w_{\text {centraldynamic }}$ is the maximum central displacement of the plate subjected to moving load. Define the dynamic coefficient as the ratio

$$
\text { Dynamic coefficient }=\frac{w_{\text {centerdynamic }}}{w_{\text {centerstatic }}}
$$

Figure 8 shows the effects of the load deceleration magnitude on the dynamic coefficient during the onset of deceleration with six cases of load deceleration magnitude: $a_{d e}=-2.5,-5.0,-7.5,-9.81,-15,-20 \mathrm{~m} / \mathrm{s}$. In this example, the coefficient of static friction and kinetic friction are $\mu_{s}=1, \mu_{k}=0.85$, respectively. From Eq. (12), the load deceleration magnitude which causes the wheel sliding is $a_{\text {sliding }}=-9.81 \mathrm{~m} / \mathrm{s}$. As to be expected, Figure 8 shows that the dynamic coefficient increases rapidly when the load starts to decelerate. Furthermore, it can be seen that when the load deceleration magnitude is smaller than $a_{\text {sliding }}$ (sliding does not occur), the maximum dynamic coefficient increases as the load deceleration magnitude increases. In contrast, when the load deceleration magnitude is larger than $a_{\text {sliding }}$ (sliding occurs), the maximum dynamic coefficient is constant as the load deceleration magnitude increases. The reason for this may be explained as due to the friction force between the wheel and pavement is constant when the sliding occurs. Consequently, the maximum dynamic coefficient of plate is constant.

Figure 9 shows the effects of the kinetic friction coefficient on the dynamic coefficient with six cases of the kinetic friction coefficient: $\mu_{k}=0,0.45,0.55,0.65$, $0.75,0.85$. In case of the kinetic friction coefficient $\mu_{k}=$ 0 , the plate is subjected to the decelerated moving load without the friction force. The load deceleration magnitude $a_{d e}=-20 \mathrm{~m} / \mathrm{s}$ (sliding occurs) is employed in this example. As to be expected, it can be seen from Figure 9 that the maximum dynamic coefficient increases as the kinetic friction coefficient increases. It can be inferred as due to the friction force increases when the kinetic friction coefficient increases. Consequently, the maximum dynamic coefficient of plate increases.

Finally, the friction moment acting on the plate caused by the friction force depends significantly on the thickness of the plate. Error! Reference source not found. shows the effects of the plate thickness on the dynamic coefficient with five cases of the plate thickness: $h=0.2$, $0.4,0.6,0.8,1.0 \mathrm{~m}$. The load deceleration magnitude is $a_{d e}=-5 \mathrm{~m} / \mathrm{s}$ (sliding does not occur). As to be expected, the dynamic coefficient increases as the plate thickness increases. However, it is noted that the dynamic coefficient increases significantly when the plate thickness increases from $0.2 \mathrm{~m}$ to $0.6 \mathrm{~m}$. In contrast, the dynamic coefficient increases lightly when the plate thickness is more than $0.6 \mathrm{~m}$. The reason for this may be explained as due to the plate thickness increases, both the flexural rigidity of the plate and the friction moment caused by the friction force acting on the plate increase. When the plate thickness increases from $0.2 \mathrm{~m}$ to $0.6 \mathrm{~m}$, the increase of the friction moment is more than the increase of the flexural rigidity of plate. The result is that the dynamic coefficient increases significantly as the plate thickness increases and vice versa.

\section{CONCLUSION}

The paper presents an extension of the one-dimensional MEM for investigated the dynamic responses of Mindlin plate resting on a viscoelastic foundation subjected to moving loads during abrupt braking. The proposed computational approach offers an effective and convenient means for calculating the dynamic responses of the pavement structure subjected to moving loads during abrupt braking and similar problems involving moving systems on a Mindlin plate. The parametric studies are conducted to determine the effects of various parameters on the dynamic responses of the plate and some conclusions can be drawn as follows:

- The accuracy of the proposed method is verified by comparing the present results with those of other methods in the literature.

- The dynamic coefficient increases rapidly as the load start to decelerate. When the load deceleration magnitude is smaller than $a_{\text {sliding }}$ (sliding does not occur), the maximum dynamic coefficient increases as the load deceleration magnitude increases. In contrast, when the load deceleration magnitude is larger than $a_{\text {sliding }}$ (sliding occurs), the maximum dynamic coefficient is constant as the load deceleration magnitude increases.

- The kinetic friction coefficient and the plate thickness have a significant effect on the dynamic coefficient. The results show that the maximum dynamic coefficient increases when the kinetic friction coefficient and the plate thickness increases.

\section{ACKNOWLEDGEMENTS}

This research is funded by Vietnam National University Ho Chi Minh City (VNU-HCM) under grant number B2017-20-01: "Development and application of Moving Element Method for dynamic problems in civil engineering structures".

\section{REFERENCES}

[1] [1] J. A. Gbadeyan and S. T. Oni, "Dynamic response to moving concentrated masses of elastic plates on a non-Winkler elastic foundation," J. Sound Vib., vol. 154, no. 2, pp. 343-358, 1992.

[2] [2] S. M. Kim and J. M. Roesset, "Moving loads on 
a plate on elastic foundation," Mov. loads a plate elastic Found., vol. 124, pp. 1010-1017, 1998.

[3] [3] M. H. Huang and D. P. Thambiratnam, "Deflection response of plate on winkler foundation to moving accelerated loads," Eng. Struct., vol. 23, pp. 1134-1141, 2001.

[4] [4] M. H. Huang and D. P. Thambiratnam, "Dynamic response of plates on elastic foundation to moving loads," J. Eng. Mech., vol. 128, no. 9, pp. 1016-1022, 2002.

[5] [5] L. Sun, "Dynamic response of Kirchhoff plate on viscoelastic foundation to harmonic circular loads," J. Appl. Mech., vol. 70, no. 9, pp. 1016-1022, 2003.

[6] [6] L. Sun, "Dynamic of plate generated by Moving Harmonic Loads," J. Appl. Mech., vol. 72, pp. 772777,2005

[7] [7] J. S. Wu, M. L. Lee, and T. S. Lai, "The dynamic analysis of a flat plate under a moving load by finite element method," Int. J. Numer. Methods Eng., vol. 124, pp. 1010-1017, 1987.

[8] [8] M. Zaman, M. R. Taheri, and A. Alvappallai, "Dynamic response of thick plate on elastic foundation to moving loads," Int. J. Numer. Anal. Methods Geomech., vol. 15, pp. 627-647, 1991.

[9] [9] P. Phung-Van, H. Luong-Van, T. H. NguyenThoi, and Nguyen-Xuan, "A cell-based smoothed discrete shear gap method (CS-DSG3) based on the higher-order shear deformation theory for dynamic responses of Mindlin plates on the viscoelastic foundation subjected to a moving sprung vehicle," Int. J. Numer. Methods Eng., vol. 98, no. 13, pp. 988-1014, 2014.

[10][10] T. Q. M. Li, Y. Zhong, and H. Zong, "Dynamic response of rectangular plate subjected to moving load with variable velocity," J. Eng. Mech., vol. 4, 2013.

S. Krenk, L. Kellezi, S.R.K. Nielsen, and P.H. Kirkegaard, "Finite elements and transmitting boundary conditions for moving loads", In: Proceedings of the 4th European Conference on Structural Dynamics, Eurodyn '99, Praha,(1999), pp. 447-452.

[11]L. Andersen, S.R.K. Nielsen, and P.H. Kirkegaard, "Finite element modelling of infinite Euler beams on Kelvin foundations exposed to moving loads in convected coordinates", Journal of Sound and Vibration, Vol. 241, No. 4, pp. 587-604, 2001.

[12]C.G. Koh, J.S.Y. Ong, D.K.H. Chua, and J. Feng, "Moving element method for train-track dynamics". International Journal for Numerical Methods in Engineering, Vol. 56, pp. 1549-1567, 2003.

[13]C.G. Koh, P.P. Sze, and T.T. Deng, "Numerical and analytical methods for in-plane dynamic response of annular disk", International Journal of Solids and Structures, Vol. 43, pp. 112-131, 2006.

[14]C.G. Koh, G.H. Chiew, and C.C. Lim, "A numerical method for moving load on continuum", Journal of Sound and Vibration, Vol. 300, pp. 126-138, 2007.

[15]W.T. Xu, J.H. Lin, Y.H. Zhang, D. Kennedy, and F.W. Williams, "2D moving element method for random vibration analysis of vehicles on Kirchhoff plate with Kelvin foundation", Latin American Journal of Solids and Structures, Vol. 6, pp. 169-183, 2006.

[16]K.K. Ang and J. Dai, "Response analysis of high-speed rail system accounting for abrupt change of foundation stiffness", Journal of Sound and Vibration. Vol. 332, pp. 2954-2970, 2013.

[17] K.K. Ang, J. Dai, M.T. Tran, and V.H. Luong, “Analysis of high-speed rail accounting for jumping wheel phenomenon". International Journal of Computational Methods, Vol. 11, 1343007 (12 pages), 2014.

[18]M.T. Tran, K.K. Ang, and V.H. Luong, "Vertical dynamic response of non-uniform motion of high-speed rails". Journal of Sound and Vibration, Vol. 333, 5427-5442, 2014.

[19]J. Dai and K.K. Ang, "Steady-state response of a curved beam on a viscously damped foundation subjected to a sequence of moving loads". Journal of Rail and Rapid Transit, Available: DOI: 10.1177/0954409714563366.[Accessed: 2015]

[20] M.T. Tran, K.K. Ang, and V.H. Luong, "Dynamic response of high-speed rails due to heavy braking". Journal of Rail and Rapid Transit, Available: http://dx.doi.org/10.1177/0954409716639997. [Accessed: 2016]

[21]M.T. Tran, K.K. Ang, and V.H. Luong, "Multiple-rail high speed train subject to braking", Journal of Structural Stability and Dynamics, Available: http://dx.doi.org/10.1142/S0219455417500717. [Accessed: 2016]

[22] M.T. Tran, K.K. Ang, and V.H. Luong, "Dynamic response of high-speed rails subject to abrupt braking", Vehicle System Dynamics: International Journal of Vehicle Mechanics and Mobility (2017) (Accepted).

[23]M.T. Tran, K.K. Ang, and V.H. Luong, "Vertical dynamic response of high-speed rails during sudden deceleration", International Journal of Computational Methods, Vol. 14, No. 1, 1750014 (24 pages), 2007, Available: DOI 10.1142/S0219876217500141.

[24] Y. Zhao, Y. Qiu, P. Cao, and C. Ai, "Research on dynamic response of pavement under moving vehicle load using three dimensional finite element method", Applied Mechanic and Materials, Vol. 444-445, pp. 1197-1203, 2014.

[25]"Wikipedia",Available: https://en.wikipedia.org/wiki/Friction\#cite_note-roymech20. 